\title{
Sexual Dysfunction: Primary, Secondary or a by-product of secret issues?
}

Roche lle Hamilton Clinical Sexologist Barwon Consulting Suites Geelong Aus tra lia

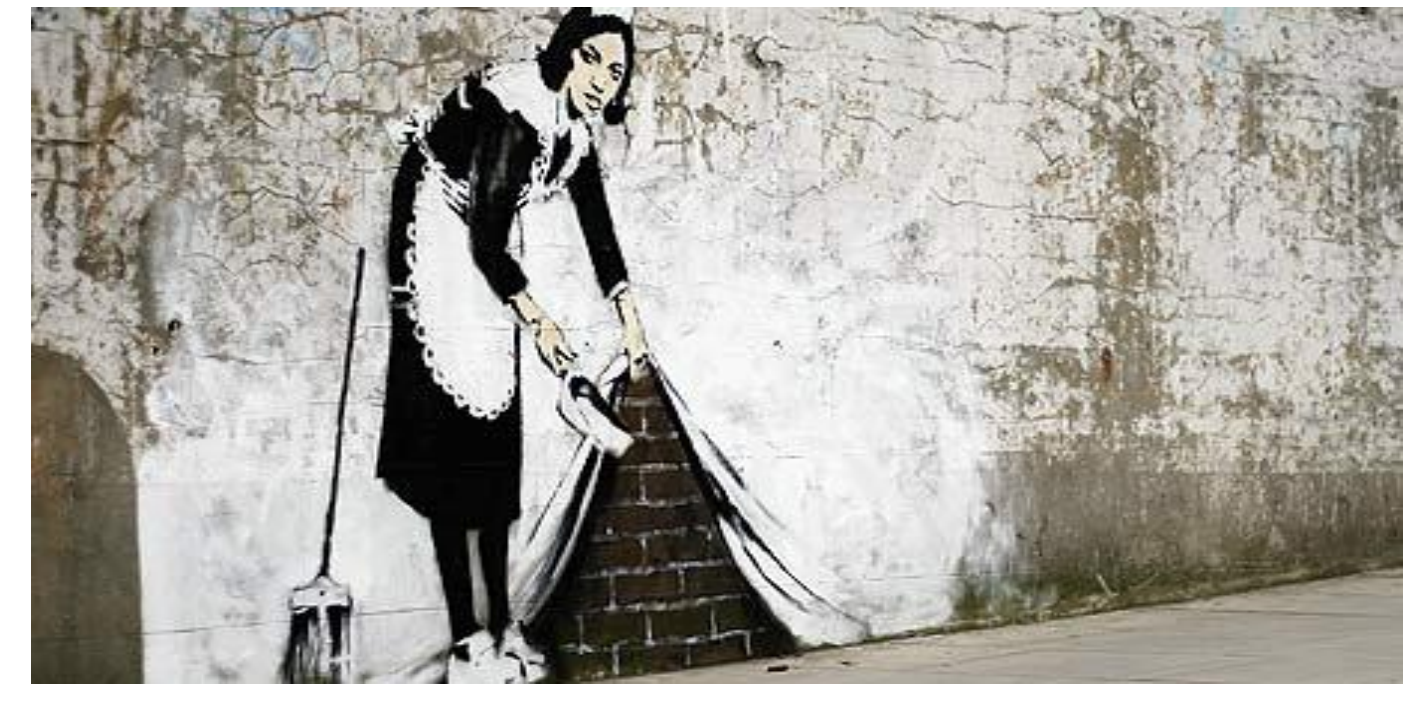

Aim: To explore the referral diagnosis of sexual dysfunction from a psychosexual basis.

Methods: A retrospective analysis of 50 women referred to a clinical sexologist for varying aspects of sexual dysfunction during January 2016 - December 2016.

Sexual dysfunction can be many things. But fundamentally it is anything that hinders, prevents or causes significant psychological, emotional or physical pain to participate or enjoy sexual intimacy.

Some reasons this may occur include:

口 Medical \& psychological implications

Co-morbidities

Awareness of the normal aging process

Overlapping variables which can mask some interpretation of dysfunction

Guilt

Results: $100 \%$ of women exhibited a variety of sexual dysfunction. $44 \%$ displayed variables of sexual abstinence not explored or discussed by the referring Health Care Professional (HCP).

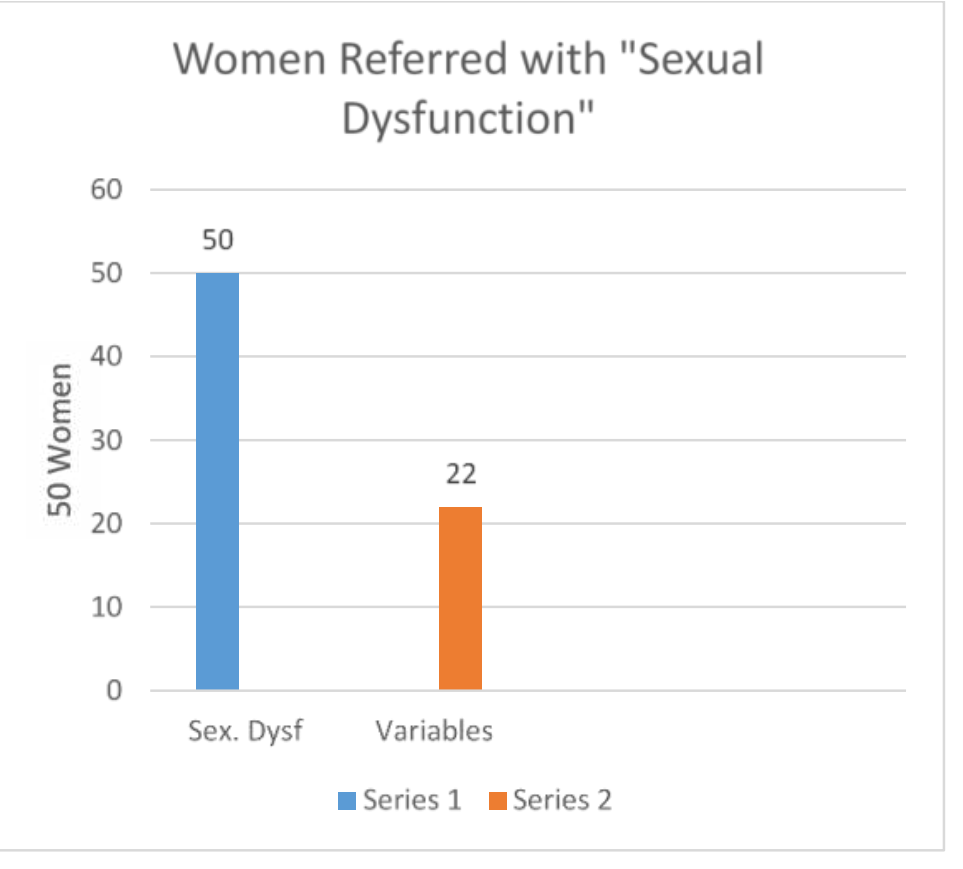

Table 1

Conclusion: Sexual Dysfunction referrals incorporate a broad range of issues which fall into sub-categories, requiring further classification as primary or secondary. It is identified that a significant proportion is incorrectly categorised by the HCP, missing the underlying true reason for sexual abstinence. The ability to inquire or frame questions within the assessment is key to unlocking the contributing or causal problem. Incorporating specific questions assists in decreasing or removing any sense of guilt the woman may have around this.

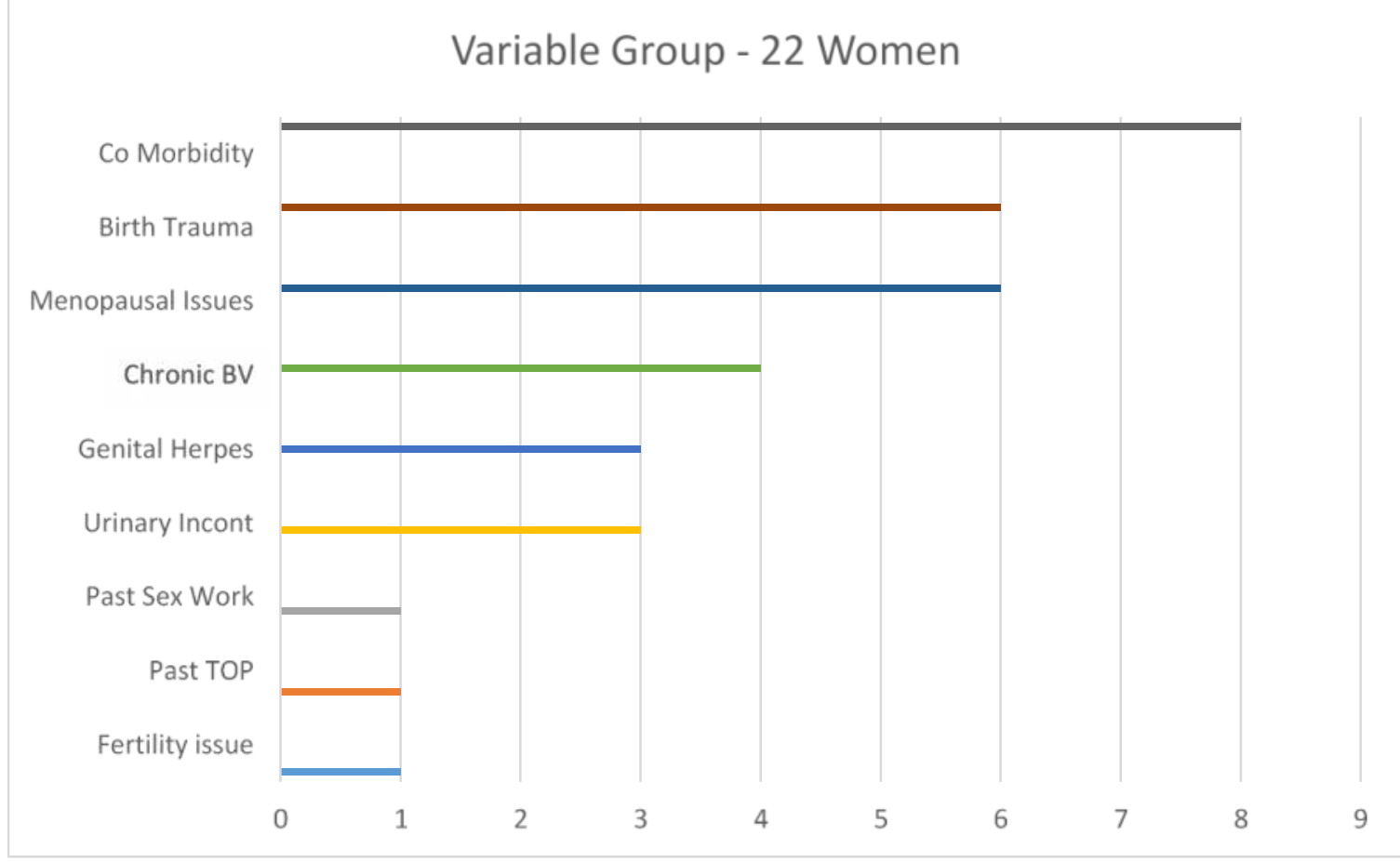

Table 2

HCPs referral diagnosed 'Sexual Dysfunction' as dyspareunia; vaginismus, female sexual desire disorder and depression. Formal investigations had not been undertaken, nor a significant sexual history taken. The following key issues MISSED by the HCPs :

- Vaginal atrophy

- Undiagnosed chronic bacterial vaginosis

- Genital herpes

- Urinary incontinence

- Post-traumatic stress disorder - post birth trauma

- History of termination of pregnancy (some women ruminate causing a sense of guilt, marked sadness, depression)

- Sub-fertility issues

- $\quad$ Past sex work

(Table 2 \& 3)

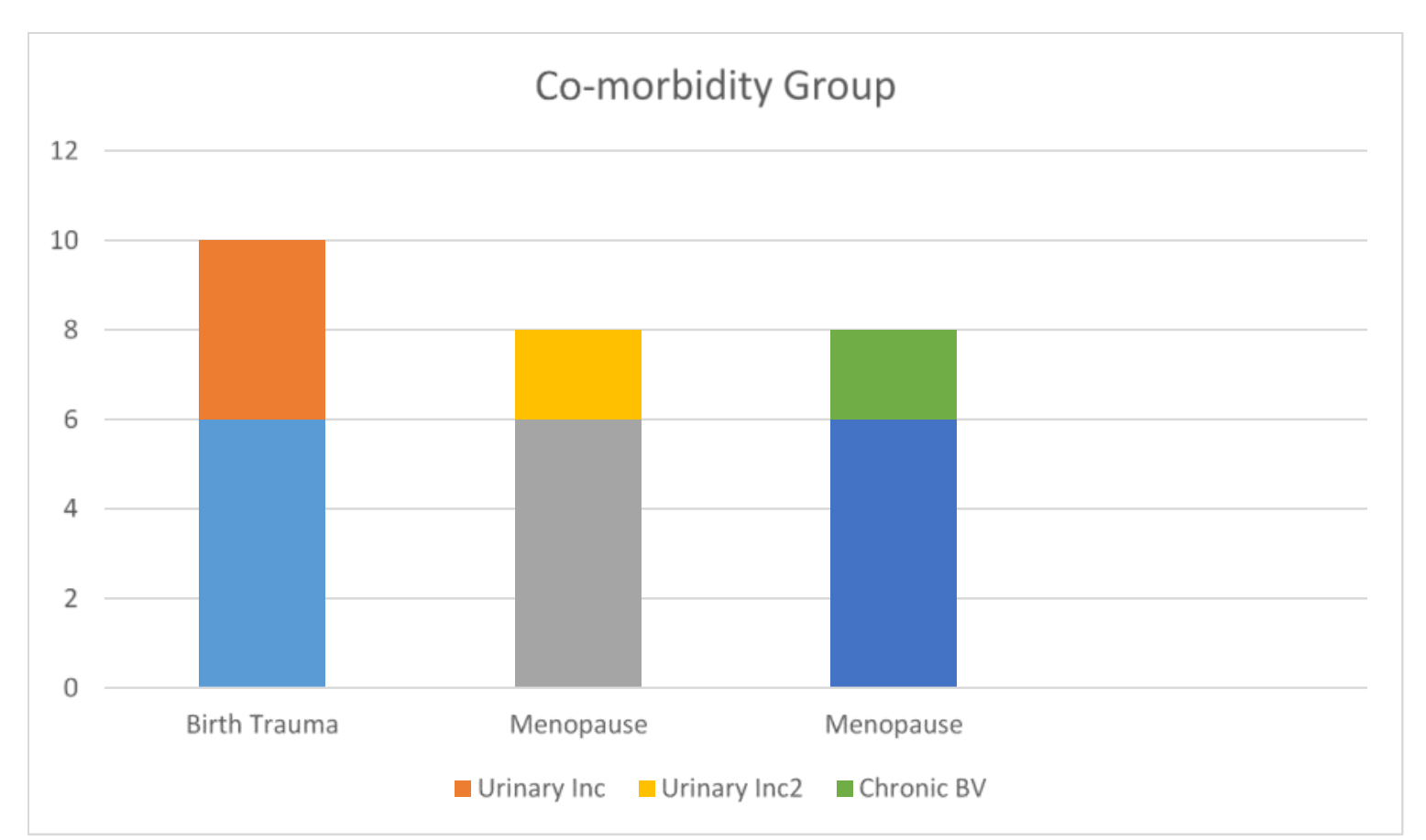

Table 3 